\section{Laminar flow of non-Newtonian shear-thinning fluids in a T-channel}

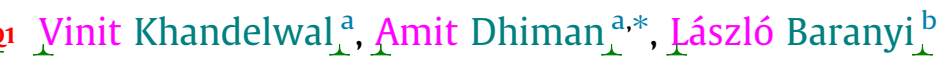

${ }^{a}$ Department of Chemical Engineering, Indian Institute of Technology Roorkee, Roorkee 247667, India

${ }^{\mathrm{b}}$ Department of Fluid and Heat Engineering, Institute of Energy Engineering and Chemical Machinery, University of Miskolc, 3515 Miskolc-Egyetemvaros, Hungary

\section{A R T I C L E I N F O}

\section{Article history:}

Received 3 July 2014

Received in revised form 30 October 2014

Accepted 26 November 2014

Available online $\mathrm{xxxx}$

\section{Keywords:}

T-channel

Non-Newtonian fluids

Laminar flow

Recirculation length

Critical Reynolds number

\begin{abstract}
A B S T R A C T
Flow characteristics of non-Newtonian power-law fluids in a right-angled horizontal T-channel are studied in the laminar regime. In particular, the two-dimensional numerical computations are performed using Ansys Fluent for the following range of physical parameters: Reynolds number $(\operatorname{Re})=5-200$ and power-law index $(n)=0.2-1$ (covering shear-thinning, $n<1$ and Newtonian, $n=1$ fluids). The flow fields have been explained by streamline contours. The engineering parameters such as wake/recirculation length, critical Reynolds number for the onset of flow separation and the variation of viscosity along the lower wall of side branch are calculated for the above range of settings by using constant density and non-Newtonian power-law viscosity model. The results showed that for a particular $n$, length of recirculation zone increases in the side branch with increasing Re. Also, it increases with decreasing $n$ for the fixed Re. The critical Reynolds number for the onset of flow separation decreases with decreasing $n$. A simple wake-length correlation is also established at different values of Re and $n$ for the range of parameters. (C) 2014 Published by Elsevier Ltd.
\end{abstract}

\section{Introduction}

Pipe networks are widely used for transportation of liquids and gases. These networks vary from a few pipes to complex assembly of very large number of pipes. In addition to pipes, the network also consists of components causing boundary layer separation due to change in the momentum of the flow. In this work, we have concentrated our attention on a very common component of a pipe network: the T-channel. The flow of Newtonian fluids in a T-channel is characterized by two separation zones: one in the branched channel, another along the left wall of the main branch at the junction, and a stagnation point near the downstream corner of the junction (Fig. 1). A separation zone can be defined as the region of recirculating fluid with very low velocities; therefore it has a strong sediment deposition potential. Continuous sediment deposition over a period of time reduces the conveyance of the channel. Thus, in engineering practice, it is essential to understand the basic characteristics of flow in separating and reattaching flows. Flow through a T-channel has a variety of engineering applications in irrigation systems, wastewater treatment, flood water driving, biomechanical applications, phase separation, oil and gas pipelines, polymer and pharmaceutical industries, and in many other areas.

\footnotetext{
* Corresponding author. Tel.: +91 1332285890 (O), +91 9410329605 (mobile). E-mail addresses: amitdfch@iitr.ernet.in, dhimuamit@rediffmail.com (A. Dhiman).
}

Flow in a T-channel for Newtonian fluids has been investigated extensively both experimentally and numerically to obtain the basic information of flow separation and reattachment phenomena in the laminar flow regime. This work aims to study twodimensional (2-D) laminar flow for non-Newtonian power-law fluids in a T-channel over a wide range of Reynolds numbers (Re). The 2-D simulations are deemed adequate to represent actual three-dimensional (3-D) situations when the aspect ratios of the ducts forming the T-channel are large, as in the experiments of Liepsch et al. [1] and Khodadadi et al. [2]. Before going into a detailed presentation and discussion of the problem under consideration, it is useful to account briefly the current status of the relevant literature focusing on Newtonian and non-Newtonian fluids.

Grace and Priest [3] presented experimental results for the division of flow at different width ratios of the branch channel orientation to the main channel. They also classified the division of flow into two regimes, with and without the appearance of local standing waves near the branch. The regime without waves corresponded to the case where the Froude numbers were relatively small, and the regime with the waves corresponded to the free overall conditions at sections downstream of the junction. Hayes et al. [4] studied the flow characteristics of a Newtonian fluid in a 2-D planar T-branch over a range of Reynolds numbers (10-800) for two different outlet boundary conditions: (i) constant exit pressure and (ii) specified flow split between the branches. They found that the fractional flow in the main duct increases with 


\section{Nomenclature}

D non-dimensionalizing length scale, $\mathrm{m}$

$I_{2}$ second invariant of the rate of deformation tensor, $\mathrm{s}^{-2}$

$L_{d} \quad$ side branch length, $\mathrm{m}$

$L_{r} \quad$ length of recirculation region, $\mathrm{m}$

$L_{1} \quad$ total length in mainstream direction, $\mathrm{m}$

$m \quad$ power-law consistency index, Pa $s^{n}$

$n \quad$ power-law index

$N_{\text {cells }} \quad$ total number of cells in the domain

$P$ pressure, $\mathrm{Pa}$

Re Reynolds number

$t$ time, $s$

$U \quad$ velocity along $X$-axis, $\mathrm{m} / \mathrm{s}$

$V \quad$ velocity along $Y$-axis, $\mathrm{m} / \mathrm{s}$

$V_{\text {avg }}$ average velocity of the fluid at inlet, $\mathrm{m} \mathrm{s}^{-1}$
$W_{b} \quad$ width of side branch, $m$

$W_{c} \quad$ width of main branch, $m$

$X_{d} \quad$ downstream length of main branch, $m$

$X_{u} \quad$ upstream length of main branch, $m$

$X \quad$ coordinate in side stream direction, $m$

$Y \quad$ coordinate in mainstream direction, $m$

\section{Greek symbols}

$\rho$ density of fluid, $\mathrm{kg} \mathrm{m}^{-3}$

$\delta \quad$ minimum grid spacing, $\mathrm{m}$

$\Delta \quad$ maximum grid spacing, $\mathrm{m}$

$\eta \quad$ power-law viscosity, Pa s

$\tau \quad$ extra stress tensor, $\mathrm{Pa}$

$\varepsilon \quad$ rate of deformation tensor, $\mathrm{s}^{-1}$ increasing Reynolds number for the case of constant exit pressures at the outlet of each branch. For the case of specified flow split at the outlet of each branches, the size and strength of the recirculation zones increase as more fluid is forced to go into the side branch. Neary and Sotiropoulos [5] presented the numerical solutions for the steady 3-D laminar flows through a $90^{\circ}$ rectangular cross-section over a range of Reynolds numbers (496-525), discharge ratios (0.23-0.64) and duct aspect ratios (1-8). They compared solutions with experimental measurements to elucidate the flow topology patterns and showed that both length and width of the separation zone decrease with increasing discharge ratio. Weber et al. [6] performed an extensive experimental study of combining flows in a $90^{\circ}$ open channel for Reynolds numbers ranging from 500 to 1000 . They provided a very broad data set comprising three velocity components, turbulence stresses, and water surface mappings.

Yanase et al. [7] investigated the laminar flow in a curved rectangular duct for a range of aspect ratios (1-12) using spectral method and found five branches of steady solutions. They also investigated the linear stability characteristics for all the steady solutions and found that one steady solution is linearly stable for most of the aspect ratio values, but two linearly stable steady solutions exist for a region of small aspect ratio and there are several intervals of aspect ratio where there is no linearly stable steady solution. Huang et al. [8] provided a comprehensive numerical

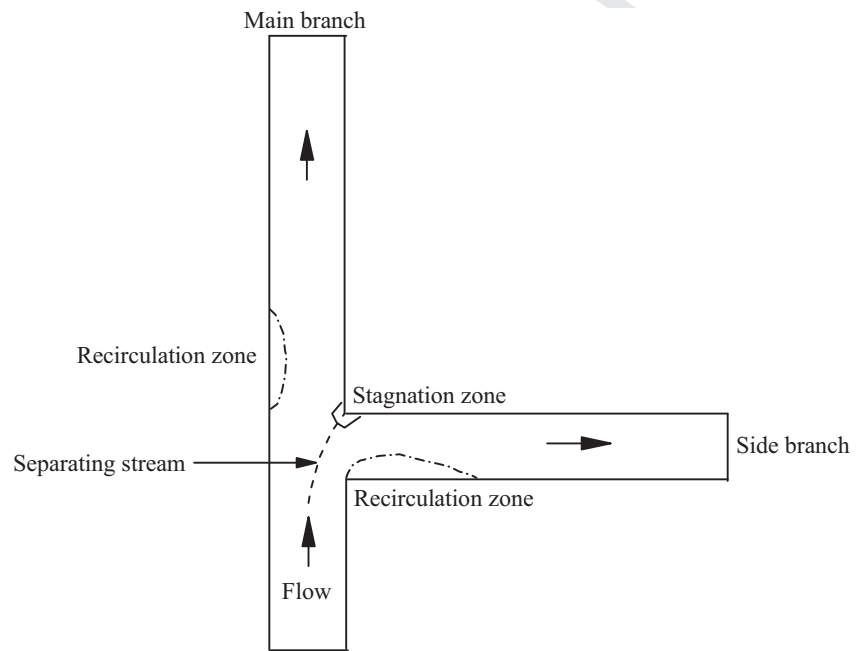

Fig. 1. Schematic of a separating flow along with recirculation and stagnation zone in a T-channel. study of combining flows in open-channel junctions using a 3-D turbulence model for varying Reynolds numbers (500-1000) and validated the model by using the detailed test data of Weber et al. [6]. Ramamurthy et al. [9] studied the open-channel laminar and turbulent 3-D flow characteristics of a $90^{\circ}$ rectangular channel junction of equal width over a parameter range of discharge ratios (0.149-0.838). They adopted the $k-\omega$ turbulence model to investigate the dividing open channel flow characteristics. They presented a data set composed of water surface mappings and 3-D velocity distributions in the vicinity of the channel junction region. Shamloo and Pirzadeh [10] investigated the characteristics of separation zones in a T-channel over a range of discharge ratios $0.2-0.8$ by using Fluent. Numerical simulations in their study were performed using the RSM turbulence model.

In addition to flow characteristics for a Newtonian fluid, Liepsch et al. [1] performed measurements and calculations for laminar flow in a plane $90^{\circ}$ bifurcation for non-Newtonian fluids for a range of Reynolds numbers (496-1130) and discharge ratios (0.23-0.64). They developed velocity profiles as a function of geometry, Reynolds number and flow rate ratio and compared it with LDA measurements. Bramley and Dennis [11] studied the 2-D steady flow for various Reynolds numbers (100, 500, 1000 and 2000) in a branching channel by writing Navier-Stokes equations in terms of stream function and vorticity, and solved them by using the difference scheme of Dennis and Hudson [12]. They presented numerical methods for dealing with the singularity in the vorticity at the sharp corners where the channel bifurcates. Ehrlich and Friedman [13] investigated the bifurcation flows extended to 2-D regions obtained from radiographs of human aortic bifurcations mapped onto rectangles with a slit. They developed correlations between computed wall vorticities and shear rates from laboratory experiments. These two quantities were proportional in the same dimensionality, suggesting that useful data can be obtained from 2-D calculations of 3-D phenomena.

Miranda et al. [14] looked at steady and periodic flows of Newtonian fluids for a range of Reynolds numbers (10-1000) and discharge ratios (0.1-0.9) through a bifurcation geometry, and obtained computational results for shear stresses in close agreement with previous experimental measurements and also with the simulations of Liepsch et al. [1], Khodadadi et al. [15] and Khodadadi [16]. Moshkin and Yambangwi [17] developed a computational method for solving viscous incompressible flow in a domain for Reynolds number ranging from 10 to 400 . They studied the effect of flow rate and curvature ratio of a planar U-bend channel and found that the size of the separation zone increases with increasing flow rate and decreasing curvature ratio. 
Miranda et al. [14] also examined a single case of non-Newtonian flow for the blood analogue fluid and left open a route of investigation dealing with the analysis of other fluid characteristics, such as the level of shear-thinning, upon the flow patterns around the bifurcation. Recently, Matos and Oliveria [18] studied the steady and unsteady flows of a 2-D planar T-junction for Reynolds number range 50-1000 (in the interval of 50) for Newtonian and non-Newtonian fluids $(n=0.1-1)$ using Carreau-Yasuda equation, one of the most utilized generalized Newtonian fluid models in blood flow simulations. In their study, they choose different flow rate ratios at the outlet boundary of the side branch to examine the flow characteristics in the T-junction. Extensive results on the eddy lengths and intensities and on the shear stress fields are reported. Conversely, the present study is dedicated to analyzing the flow characteristics of Newtonian and non-Newtonian fluids $(n=0.2-$ 1) using power-law model in the lower Reynolds number range $(<200)$ by assuming both of the exits to be at the same pressure (atmospheric pressure), which is consistent with the first case of boundary condition of Hayes et al. [4]. It is also worthwhile to mention that non-Newtonian effects are more prominent at low values of Re and still no information is available for non-Newtonian fluids at such low Re, especially up to $R e=50$. A correlation for the wake length and the determination of the onset of flow separation are the unique aspects of the present investigation.

Some studies on the use of power-law model are also worth mentioning at this stage. Hajmohammadi et al. [19] studied the effects of a thin gas layer on the hydrodynamic aspects of powerlaw fluids $(n=0.15-1.4)$ in a radial Couette flow between two cylinders. They gave an analytical solution to determine the velocity profile in the two-phase flow system. It was shown that the thin (micro) gas layer contributes in reducing torque to set the fluid in motion in the case of Newtonian and shear-thickening fluids, but in the case of shear-thinning fluids a slight increase in the torque (about 6\%) is observed. In a similar study, Hajmohammadi and Nourazar [20] analyzed the influence of a thin gas layer (into a cylindrical Couette flow assembly) dealing with a power-law liquid $(0.1<n<1.4)$ on the torque required for activating the lubrication process, on the maximum temperature of the shaft (inner cylinder) and on Nusselt number of the two-phase flow configuration. Results showed that the thin gas layer contributes to reduce in the torque to set the fluid in motion and to downscale the maximum temperature at the shaft for Newtonian and shear-thickening liquids. However, for shear-thinning liquids, the above-mentioned positive roles break down for a large number of flow configurations. Eesa and Barigou [21] studied numerically the effects of a superimposed sinusoidal rotational vibration on the flow of nonNewtonian fluids in a tube. They found that Newtonian flow is unchanged by any superimposed oscillations but the flow of shear-thinning and visco-plastic fluids is enhanced, whilst the flow of shear-thickening fluids is retarded. Turkyilmazoglu [22] studied the magneto-hydrodynamic slip flow of an electrically conducting, non-Newtonian fluid past a shrinking sheet and found that the presence of a magnetic field has substantial effects on velocity and temperature fields. Hu and Kieweg [23] studied the effect of surface tension on the gravity-driven thin film flow of Newtonian and shear-thinning fluids $(n=0.5-1)$ and showed that capillary ridge height increased with higher surface tension, steeper inclination angle, larger initial thickness and also with decreasing shearthinning behavior.

Unlike [19,20], Hajmohammadi et al. [24] studied laminar, viscous and incompressible fluid flow configuration of bend tubes for optimizing the heat and fluid flow for varying Reynolds (500, 1652 and 2000) and Prandtl (0.7, 7 and 6780) numbers. They showed that the pressure drop and the entropy generation are considerably reduced when implementing the optimum layout, compared to the case of a fully curved tube section. Csizmadia and Hős [25] reported the estimation of loss coefficients for typical pipeline diffusers and elbows in the case of Bingham and power-law fluids. Diffusers with angle range $7.5_{-}-40^{\circ}$ and elbows of curvature radius to diameter ratio range $1-10$ were studied. They found that the standard $k-\varepsilon$ model fails to predict the flow field accurately whereas the eddy-viscosity SST turbulence model and the BSL Reynolds-stress model give very close results, and they suggest the use of the SST model.

Thus, based upon the above discussion, it can be summarized here that most of the currently available literature on Newtonian and non-Newtonian flow through a T-channel is for high Reynolds numbers, where the main thrust has been to investigate the wake phenomenon. However, in spite of having the many engineering applications mentioned above, the non-Newtonian flow through a T-channel has not been investigated in the literature at low Reynolds numbers. Therefore, the main objective of this study is to investigate the characteristics of non-Newtonian power-law flow for the following range of conditions: Reynolds number $(\mathrm{Re})=5$ 200 and power-law index $(n)=0.2-1$ (covering shear-thinning, $n<1$ and Newtonian, $n=1$ fluids). The other important objective of this study is to determine the critical Reynolds numbers at which the onset of flow separation occurs for Newtonian and shear-thinning fluids.

\section{Problem statement and mathematical formulation}

The laminar flow of non-Newtonian shear-thinning fluids through a 2-D T-channel is schematically displayed in Fig. 2. The channel inlet of width $W_{c}=D$ (also the non-dimensionalizing length scale) is exposed to a fully developed velocity field with an average velocity of $V_{\text {avg }}$ at the channel inlet (also the non-dimensionalizing velocity scale). The non-dimensional upstream distance between the inlet plane and the junction of the channel $\left(X_{u} / D\right)$ is taken as 10 , and the downstream distance

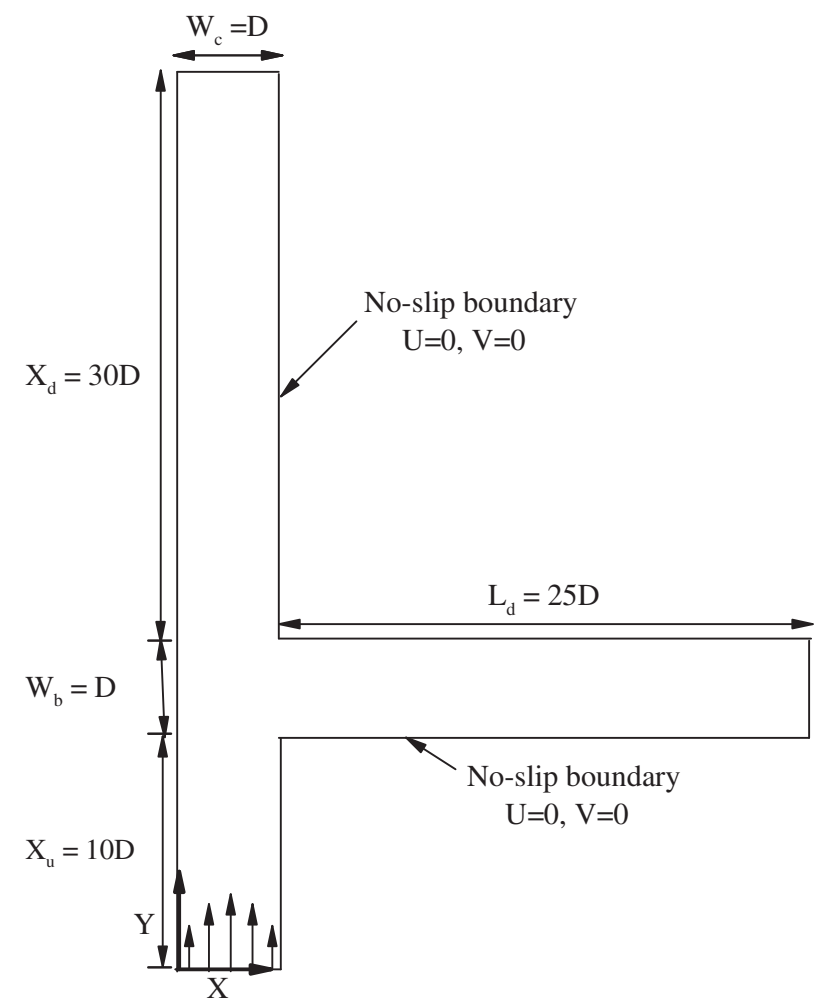

Fig. 2. Schematic diagram of flow in a T-channel. 
between the junction and the exit plane $\left(X_{d} / D\right)$ is taken as 30 with the total length of the computational domain being $L_{1} / D=41$ in the mainstream direction. The non-dimensional length of side branch $\left(L_{d} / D\right)$ is taken as 25 . These length ratios were chosen in the following manner.

In order to explore the influence of the assumed finite domain, especially for the largest Reynolds number, computations were carried out at $\operatorname{Re}=200$ for both Newtonian and non-Newtonian fluids and dimensionless side branch lengths of 20,25, 30. The influence of the side branch length $\left(L_{d} / D\right)$ on the length of the recirculation zone is presented in Table 1 . The relative difference in the reattachment length for length ratios of 25 and 30 was found to be about 3.2\%, while for the case of maximum shear-thinning behavior $(n=0.2)$ studied, the relative difference in the reattachment length for the length ratios of 25 and 30 was found to be about $3.7 \%$. This was observed to be small enough to justify the choice of a side branch length ratio of 25 for further simulations. Similarly, the variation in the reattachment length for the downstream lengths $\left(X_{d} / D\right)$ of 25,30 and 35 at $\mathrm{Re}=200$ is presented in Table 2 . The relative difference in reattachment length for 30 and 35 is found to be about $1.7 \%$, while for the case of maximum shear-thinning behavior, it was found to be about 3.6\%. Hence, a dimensionless downstream length of 30 is selected for further simulations. Likewise, the variation in the reattachment length for the dimensionless upstream lengths $\left(X_{u} / D\right)$ of 5,10 and 15 at $\operatorname{Re}=200$ is presented in Table 3 and the corresponding difference in reattachment length for 10 and 15 is found to be less than $0.4 \%$, while for the case of maximum shear-thinning behavior, it was found to be about $1 \%$. Thus, a dimensionless upstream length of 10 has been used in all the subsequent numerical calculations.

The following assumptions have been made in this study: the 2D, incompressible, isothermal and laminar flow of shear-thinning fluids. Also, the gravity, buoyancy and viscous dissipation effects are assumed negligible.

Under the above assumptions, the continuity, the $X$ and $Y$ components of Cauchy's equations in their dimensional form are known as [26,27].
Continuity:

$\frac{\partial U}{\partial X}+\frac{\partial V}{\partial Y}=0$

$X$ Momentum:

$\frac{\partial U}{\partial t}+U \frac{\partial U}{\partial X}+V \frac{\partial U}{\partial Y}=-\frac{\partial P}{\partial X}+\frac{1}{\operatorname{Re}}\left(\frac{\partial \tau_{X X}}{\partial X}+\frac{\partial \tau_{Y X}}{\partial Y}\right)$

$Y$ Momentum:

$\frac{\partial V}{\partial t}+U \frac{\partial V}{\partial X}+V \frac{\partial V}{\partial Y}=-\frac{\partial P}{\partial Y}+\frac{1}{\operatorname{Re}}\left(\frac{\partial \tau_{Y X}}{\partial X}+\frac{\partial \tau_{Y Y}}{\partial Y}\right)$

For an incompressible fluid, the extra stress tensor (which is symmetric, $\tau_{i j}=\tau_{j i}$ ) is related to the components of the rate of deformation tensor $\left(\varepsilon_{i j}\right)$ as

$\tau_{i j}=2 \eta \varepsilon_{i j}$

The components of the rate of strain tensor are related to the velocity components in Cartesian coordinates as follows:

$\varepsilon_{X X}=\frac{\partial U}{\partial X} ; \quad \varepsilon_{Y Y}=\frac{\partial V}{\partial Y} ; \quad \varepsilon_{X Y}=\varepsilon_{Y X}=\frac{1}{2}\left(\frac{\partial U}{\partial Y}+\frac{\partial V}{\partial X}\right)$

For a power-law fluid, the viscosity $(\eta)$ is defined as

$\eta=m\left(\frac{I_{2}}{2}\right)^{\frac{n-1}{2}}$

and the second invariant of the rate of deformation tensor $\left(I_{2}\right)$ is given by

$I_{2}=\sum \sum \varepsilon_{i j} \varepsilon_{j i}=2\left(\varepsilon_{X X}^{2}+\varepsilon_{X Y}^{2}+\varepsilon_{Y X}^{2}+\varepsilon_{Y Y}^{2}\right)$

where $m$ is the power-law consistency index which is a measure of the viscosity of the fluid and $n$ is the power-law index. Clearly, $n=1$ denotes the standard Newtonian flow behavior; $n<1$ corresponds to shear-thinning fluid behavior and the values of $n$ as low as $0.2-0.3$ are quite frequent for polymeric systems and particulate slurries [27]. Spelt et al. [28] also stated that shear-thinning fluids

Table 1

Influence of side branch length $\left(L_{d} / D\right)$ on recirculation region length $\left(L_{r} / D\right)$ at $\operatorname{Re}=200$.

\begin{tabular}{|c|c|c|c|c|c|}
\hline \multirow[t]{2}{*}{$L_{d} / D$} & \multirow[t]{2}{*}{$N_{\text {cells }}$} & \multicolumn{2}{|l|}{$n=1$} & \multicolumn{2}{|l|}{$n=0.2$} \\
\hline & & $L_{r} / D$ & \% Relative difference & $L_{r} / D$ & \% Relative difference \\
\hline 20 & 149,625 & 4.0708 & 7.1 & 9.2883 & 7.6 \\
\hline 25 & 154,875 & 3.9233 & 3.2 & 8.9567 & 3.7 \\
\hline 30 & 160,125 & 3.8013 & & 8.6353 & \\
\hline
\end{tabular}

Table 2

Influence of downstream length $\left(X_{d} / D\right)$ on recirculation region length $\left(L_{r} / D\right)$ at $\operatorname{Re}=200$.

\begin{tabular}{|c|c|c|c|c|c|}
\hline \multirow[t]{2}{*}{$X_{d} / D$} & \multirow[t]{2}{*}{$N_{\text {cells }}$} & \multicolumn{2}{|l|}{$n=1$} & \multicolumn{2}{|l|}{$n=0.2$} \\
\hline & & $L_{r} / D$ & \% Relative difference & $L_{r} / D$ & \% Relative difference \\
\hline 25 & 149,625 & 3.8033 & 4.7 & 8.5137 & 8.2 \\
\hline 30 & 154,875 & 3.9233 & 1.7 & 8.9567 & 3.5 \\
\hline 35 & 160,125 & 3.9910 & & 9.2782 & \\
\hline
\end{tabular}

Table 3

Influence of upstream length $\left(X_{u} / D\right)$ on recirculation region length $\left(L_{r} / D\right)$ at $\operatorname{Re}=200$.

\begin{tabular}{|c|c|c|c|c|c|}
\hline \multirow[t]{2}{*}{$X_{u} / D$} & \multirow[t]{2}{*}{$N_{\text {cells }}$} & \multicolumn{2}{|l|}{$n=1$} & \multicolumn{2}{|l|}{$n=0.2$} \\
\hline & & $L_{r} / D$ & \% Relative difference & $L_{r} / D$ & \% Relative difference \\
\hline 5 & 149,625 & 3.9418 & 0.9 & 9.1338 & 3.0 \\
\hline 10 & 154,875 & 3.9233 & 0.4 & 8.9567 & 1.0 \\
\hline 15 & 160,125 & 3.9080 & & 8.8657 & \\
\hline
\end{tabular}


are much more common than shear-thickening fluids $(n>1)$, with usually $n \geqslant 0.2$. Thus, the range of values of the power-law index $0.2 \leqslant n \leqslant 1$ used here is guided by the behavior of industrially important fluids. Furthermore, as one reduces the value of powerlaw index then it is sometimes difficult to achieve the same level of convergence because of the highly non-linear nature of the governing equations [28-34]. Because of these facts, in the literature it is very rare to use the value of power-law index smaller than 0.2 for power-law fluids and it seems to have become standard to begin at $n=0.2$ with power-law fluids.

The only dimensionless group appearing in Eqs. (2) and (3) is the Reynolds number, which is defined in this study as follows based on [27-34].

$\operatorname{Re}=\frac{\rho D^{n} V_{a v g}^{2-n}}{m}$

Needless to say that this form of Re becomes the common Newtonian form for $n=1$. One can obtain the average velocity $\left(V_{a v g}\right)$ from the flow rate using continuity.

The boundary conditions in their dimensionless form for this flow configuration (see Figs. 1 and 2) may be written as follows:

- At the inlet boundary: the fully developed velocity profile for the laminar flow of power-law fluids in a channel is given as [34],

$$
U=0 \text { and } V=\left(\frac{2 n+1}{n+1}\right) V_{\text {avg }}\left[1-\left(\left|1-\frac{2 X}{D}\right|\right)^{\frac{n+1}{n}}\right] \text { for }(0 \leqslant X \leqslant D)
$$

- At the left and right boundary walls of main branch:

$U=0$ and $V=0$ (no-slip condition)

- At the upper and lower walls of side branch:

$U=0$ and $V=0$ (no-slip condition)

- At the outlet boundary of main and side branches: the default pressure outlet condition (both exits exposed to atmosphere) available in Ansys Fluent is used, that is, zero gauge pressure at both the exits.

\section{Numerical solution procedure}

The computational grid structure adopted for solving the flow in a T-channel is depicted in Fig. 3. The grid is generated by using Gambit, and shows the non-uniform orthogonal grid structure for the whole of the computational domain (Fig. 3a) along with its magnified view (Fig. 3b). It consists of both uniform and non-uniform grid distributions with a close clustering of grid points in the regions of large gradients and coarser grids in the regions of low gradients. The junction of the channel where the flow diversion

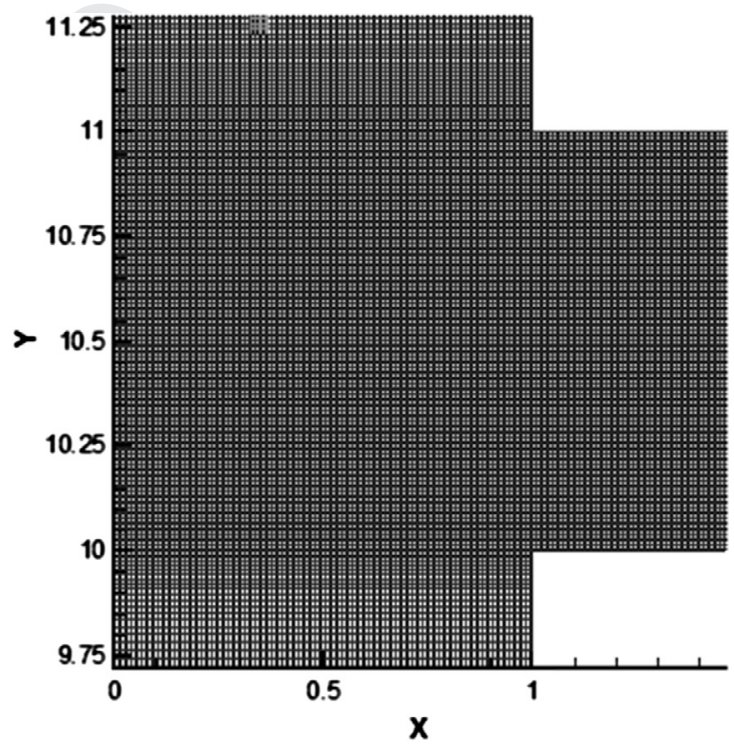

(b)

Fig. 3. Non-uniform grid structure for the flow in a T-channel: (a) complete and (b) magnified views.

Table 4

Grid sensitivity analysis for the flow through a T-channel at different values of Re and $n$.

\begin{tabular}{|c|c|c|c|c|c|c|c|c|}
\hline \multicolumn{3}{|c|}{ Grid details } & \multicolumn{6}{|l|}{$L_{r} / D$} \\
\hline Grid & $N_{\text {cells }}$ & $\Delta / D$ & $\operatorname{Re}=50$ & \% Relative difference & $\operatorname{Re}=100$ & \% Relative difference & $\operatorname{Re}=200$ & \% Relative difference \\
\hline \multicolumn{9}{|l|}{$n=1$} \\
\hline G1 & 106,500 & 0.01 & 1.8932 & 0.10 & 2.6649 & 0.07 & 3.9217 & 0.05 \\
\hline G2 & 154,875 & 0.008 & 1.8951 & 0.01 & 2.6665 & 0.01 & 3.9233 & 0.01 \\
\hline G3 & 254,007 & 0.006 & 1.8952 & & 2.6669 & & 3.9237 & \\
\hline \multicolumn{9}{|c|}{$n=0.2$} \\
\hline G1 & 106,500 & 0.01 & 3.2238 & 2.20 & 5.5139 & 1.92 & 8.8623 & 2.35 \\
\hline G2 & 154,875 & 0.008 & 3.2704 & 0.80 & 5.5826 & 0.70 & 8.9567 & 1.30 \\
\hline G3 & 254,007 & 0.006 & 3.2964 & & 5.6217 & & 9.0742 & \\
\hline
\end{tabular}


takes place is a critical zone where considerable gradients in velocity components will occur. For handling this situation, a very fine grid is used in this zone. In order to capture the physics of the boundary layer separation in the side branch a very fine grid has been constructed over a distance of $5 D$. The rest is a non-uniform grid progressively increasing in the direction away from the junction in both upstream and downstream directions, such that the smallest control volumes are in the vicinity of the junction and the largest control volumes are away from the junction.

It is important to investigate the effect of grid size on the physical output parameters. A thorough analysis of the sensitivity of the simulation results with regard to the number of elements and the grid fineness for each case was carried out using three different grids with 106,500, 154,875 and 254,007 cells of varying fineness and the results are presented in Table 4 . The minimum grid spacing $(\delta)$ at the junction and in the vicinity of the junction along the side branch for the three grids is $0.01 D, 0.008 D$ and $0.006 D$, respectively, and the maximum grid spacing of $\Delta=0.5 D$ at distance far off from the junction is found to be sufficiently fine to resolve the flow characteristics in a T-channel. The first length function has also been used for stretching the cell sizes between these limits of $\delta$ and $\Delta$ in both $X$ and $Y$ directions.

The percentage relative differences in the values of reattachment lengths at the extreme values of power-law indices $(0.2$ and 1$)$ for the various values of $\operatorname{Re}(50,100$ and 200) and the three grids (106,500, 154,875 and 254,007 cells) are also mentioned in Table 4. Briefly, for the case of maximum shear-thinning behavior $(n=0.2)$ at $\operatorname{Re}=50$, the relative difference in the value of reattachment length is found to be about $0.8 \%$ for the grid with 154,875 cells as compared to the values at the grid with 254,007 cells. Similarly, at $\operatorname{Re}=200$, the corresponding relative difference for the case of $n=0.2$ in the value of reattachment length is found to be about $1.3 \%$ for the grid with 154,875 cells as compared to the values at the grid with 254,007 cells. Hence, because the difference between the values of reattachment lengths at the grids with 154,875 and 254,007 cells is reasonably small, the optimized grid size of 154,875 cells is used for further computations in order to reduce computational load (CPU time) without losing accuracy.

This numerical study has been carried out using the commercial software package Ansys Fluent based on the finite volume method. The two-dimensional, laminar, segregated solver is used to solve the incompressible flow on the collocated grid arrangement. The constant density and the non-Newtonian power-law viscosity model are used for the simulations. The second order upwind scheme is used to discretize convective terms of momentum equations. The semi-implicit method for the pressure linked equations (SIMPLE) scheme is used for solving the pressure-velocity decoupling. Ansys Fluent solves the system of algebraic equations using the Gauss-Siedel point-by-point iterative method in conjunction with the algebraic multi-grid (AMG) method. The use of AMG scheme can greatly reduce the number of iterations (and thus, $\mathrm{CPU}$ time) required to obtain a converged solution, particularly when the model contains a large number of control volumes. The absolute convergence criteria of $10^{-10}$ for the continuity, and $X$ and $Y$-components of the velocity are prescribed in the steady regime. For detailed investigation of flow characteristics in the Tchannel, unsteady simulations have also been run for the extreme values of $\operatorname{Re}$ and $n$ (i.e., $\operatorname{Re}=200, n=1$ and 0.2 ) with the absolute convergence criteria of $10^{-20}$ each for the continuity, and $X$ - and $Y$-components of the velocity.

\section{Results and discussion}

Inspection of the foregoing analysis indicates that the flow characteristics in the present system depend on five parameters. These

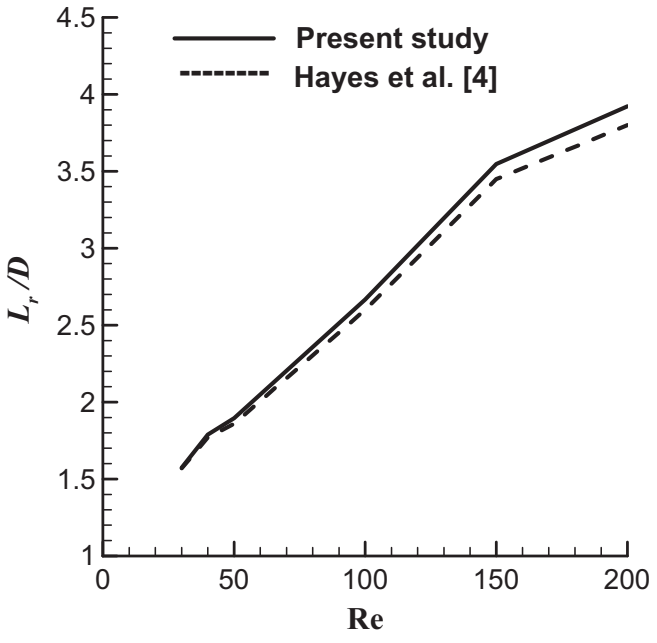

Fig. 4. Comparison of reattachment length $\left(L_{r} / D\right)$ with that of Hayes et al. [4] for $n=1$ at different values of Re.

are the Reynolds number (Re), the flow behavior index $(n)$, the dimensionless width of the main channel $\left(W_{c} / D\right)$, the dimensionless width of the side branch $\left(W_{b} / D\right)$ and the flow split or the discharge ratio. Since the vast numbers of governing dimensionless parameters are required to characterize this flow system, a comprehensive analysis of all combinations of problems is not practical. While computations can be performed for any combination of these parameters, the objective here is to present a sample of results to illustrate the effects of Re and $n$ on the flow characteristics in a T-channel. In particular, fluid flowing through the channel with $W_{c}=W_{b}=D$ is considered here. The results are presented for Reynolds number up to 200 and for $n=0.2,0.4,0.6$ and 1, thereby covering both shear-thinning and Newtonian behaviors. Physically, one can expect the centrifugal forces to induce instabilities in the 2-D flow beyond a threshold Reynolds number (more precisely the Dean number) [35], making the flow structure 3-D, even for a planar geometry. This kind of instability is more dominant in curved pipes and channels as the centrifugal force is sustained in those geometries. Although centrifugal force is not much dominant under a $90^{\circ}$ branched T-channel, but the fluid experience this force at the junction when the fluid is forced to enter the branch. The case of $n=0.8$ has not been taken up in this study because the behavior of fluid for this case is almost the same as that for Newtonian fluids. The non-Newtonian behavior is more predominant at power-law index $(n)$ values considerably less than unity.

\subsection{Validation of results}

The numerical solution procedure used here has been benchmarked with standard results for the incompressible flow of Newtonian fluids in a T-channel. The validation of reattachment length for Newtonian fluids on varying Reynolds numbers is presented in Fig. 4. The minimum percentage deviation of the values obtained in this study as compared with those given by Hayes et al. [4] is found to be about $0.23 \%$; whereas the maximum deviation is around $3.24 \%$. While generally the correspondence between the two sets of results is seen to be satisfactory, except for higher Reynolds number $(\operatorname{Re}=200$, for instance) where the two results seem to differ by about $3.24 \%$. This difference is believed to be due to the differences in the domain and/or grid sizes used in these two studies. Hayes et al. [4] used a finite element discretization on a rather coarse uniform staggered grid with a non-dimensional spacing of 0.143 , without any clustering of grid points near the junction of the channel. Thus, there are fewer control volumes at the junction 

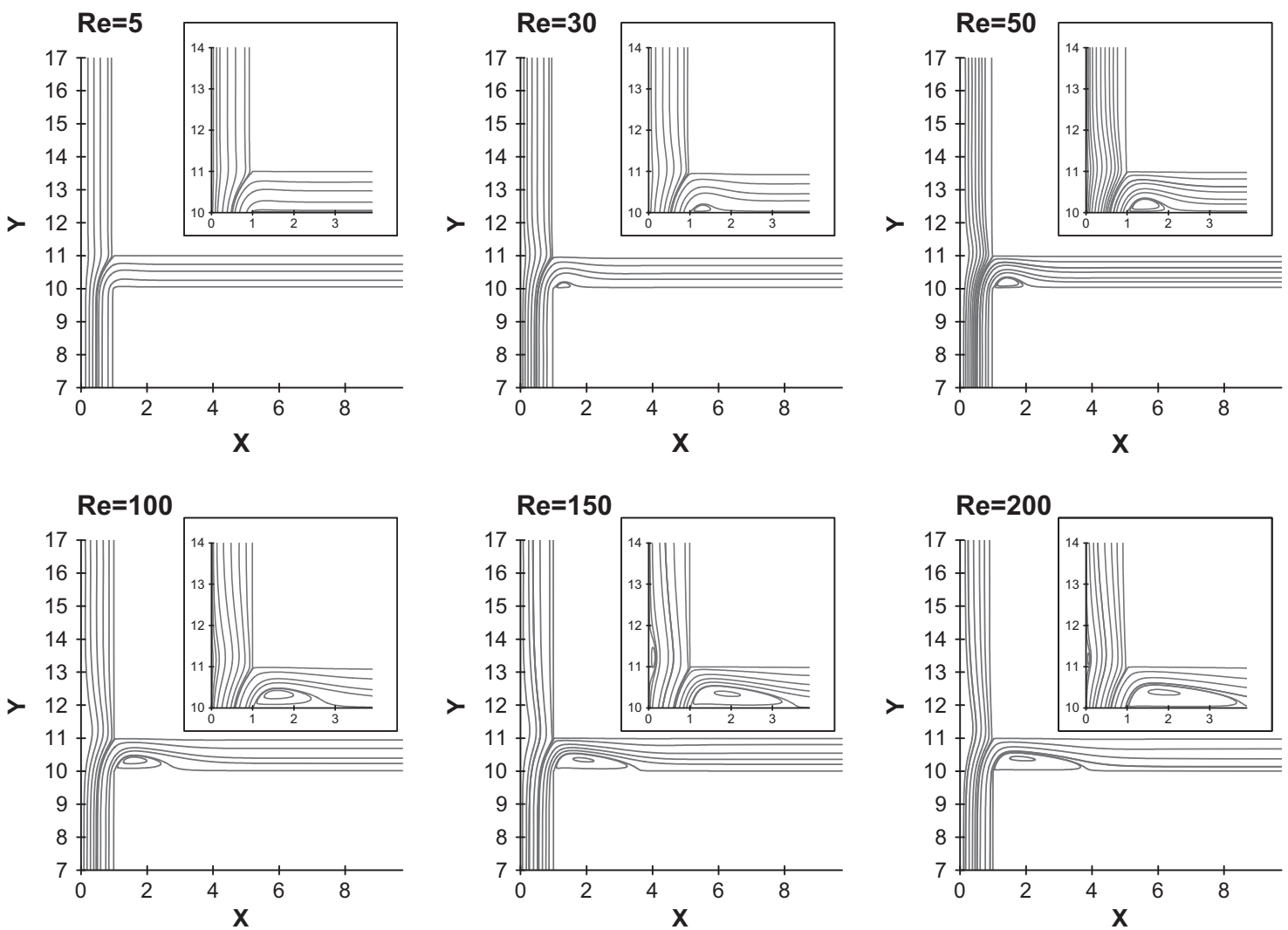

Fig. 5. Stream function contours in a T-channel at different values of Re for $n=1$.
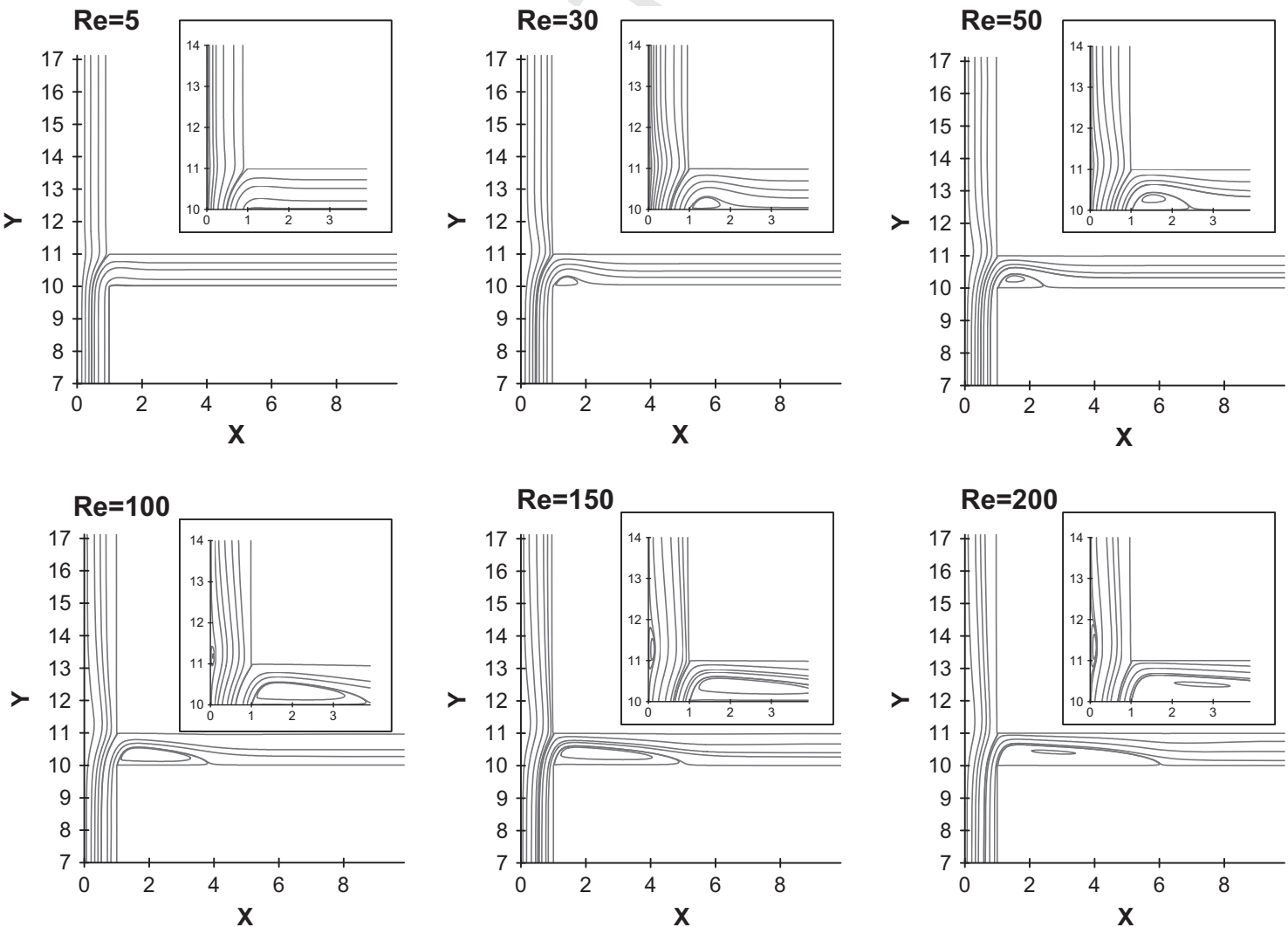

Fig. 6. Stream function contours in a T-channel at different values of Re for $n=0.6$. 

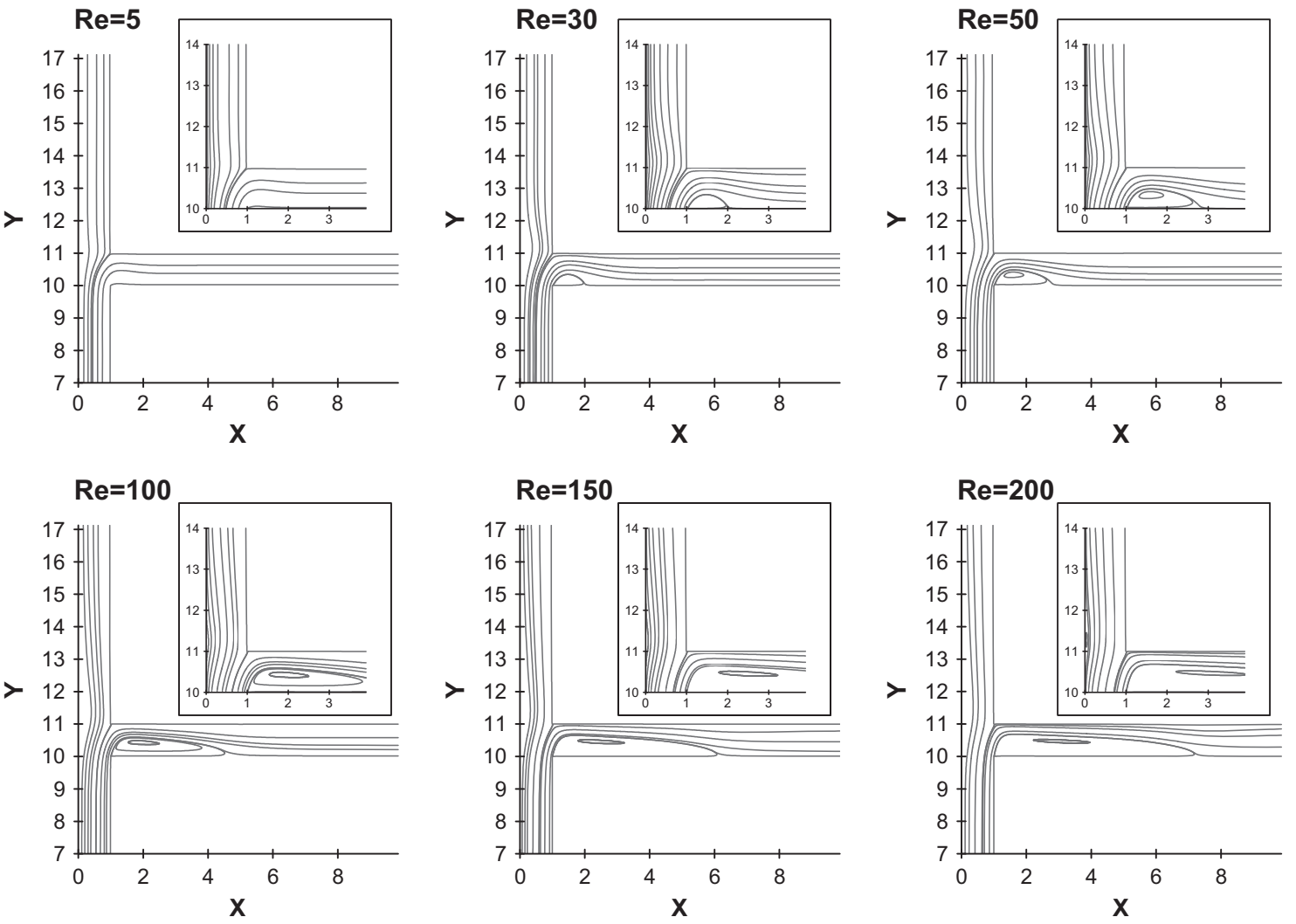

Fig. 7. Stream function contours in a T-channel at different values of Re for $n=0.4$.
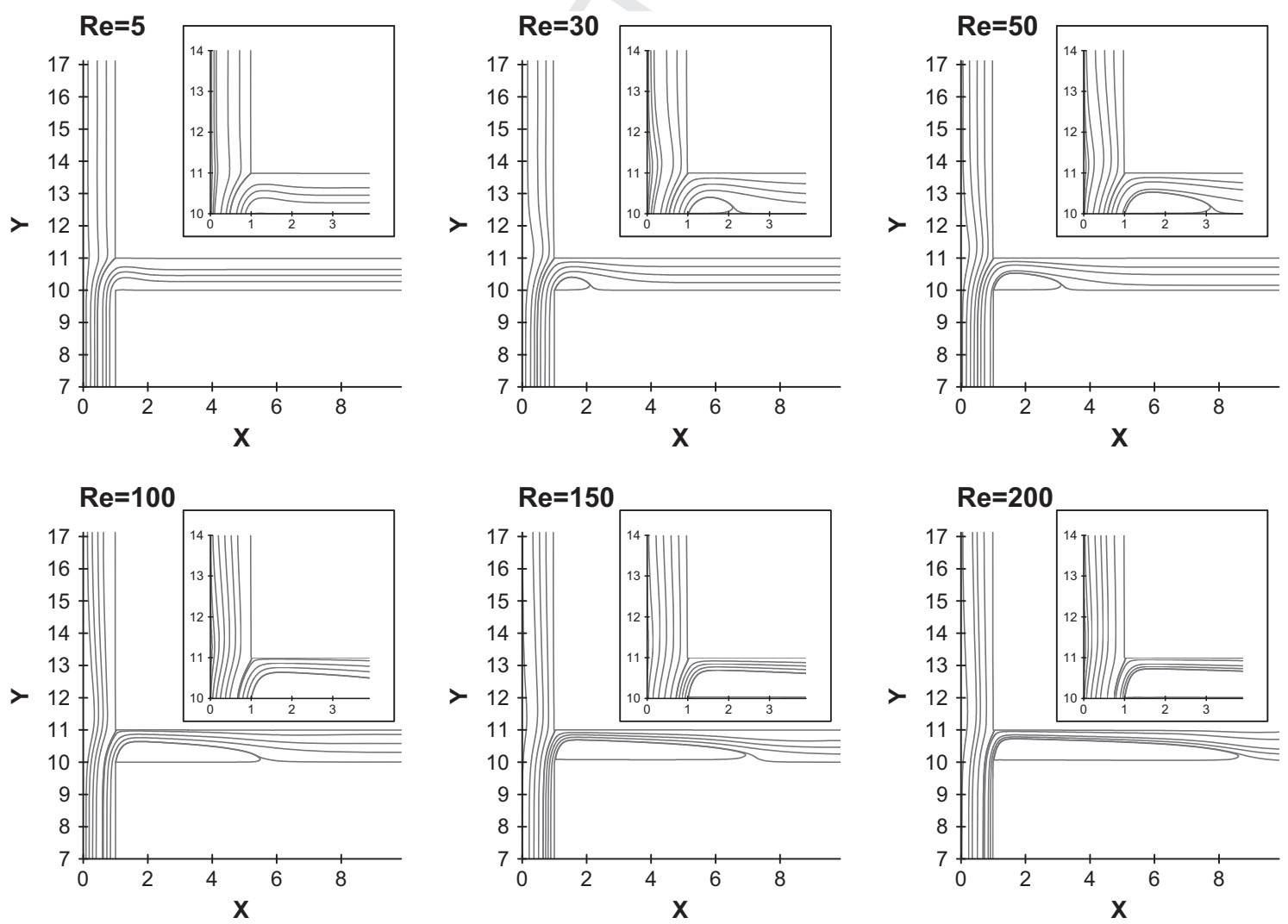

Fig. 8. Stream function contours in a T-channel at different values of Re for $n=0.2$. 
in their study as opposed to the very fine grid at the junction in the present study with a non-dimensional grid spacing of 0.008 . In fact, the differences of this order are not at all uncommon in such numerical studies [36]. Furthermore, the general validity of the code has been checked for the critical Reynolds number at which the onset of flow separation takes place for Newtonian fluids in a T-channel. The flow separation from the bottom wall of the side branch is seen to begin at $R e=17$ for the case of Newtonian fluids. A similar experimental study was conducted by Karino et al. [37] and Karino and Goldsmith [38] and their results showed the onset of flow separation in the side branch of a T-channel occurs at $\mathrm{Re}=18$. Thus, the results obtained in the current study are found to be in excellent agreement with the results available in the literature. This further confirms the accuracy and reliability of the present numerical solution procedure.

\subsection{Flow patterns}

Figs. 5-8 present the representative flow patterns by way of streamlines in the vicinity of the junction of the T-channel for $\operatorname{Re}=5,30,50,100,150$ and 200, respectively. To ensure a comparative study of the effect of the power-law index $(n)$ on the flow characteristics, four cases $(n=1,0.6,0.4$ and 0.2$)$ are presented over the entire range of Reynolds number considered. It is worthwhile to mention here that the flow remains steady for the range of settings discussed in this work. Fig. 5 shows the flow patterns of Newtonian fluids in a T-channel. For the case of Newtonian fluids, no recirculation zone is seen to occur in the side branch till $\mathrm{Re}=16$. The fluid while travelling from main branch to side branch maintains contact with the wall of the side branch. As the Reynolds number is increased, beyond a critical point the fluid gets separated from the lower wall of the side branch and a closed recirculation region is developed. The discussion on the onset of flow separation in the side branch at different values of $n$ is given in Section 4.4. Further downstream of the reattachment points, the flow regains its fully developed flow behavior. With a gradual increase in the value of the Reynolds number ( $\mathrm{Re}>16$ ), the size of recirculation region increases. The flow patterns for the case of Newtonian fluids in a T-channel are found to be in close agreement with those of Hayes et al. [4] and Neary and Sotiropoulos [5].

For the case of shear-thinning fluids, no recirculation zone is seen to occur in the side branch till $\operatorname{Re}=13,11$ and 8 for the cases of $n=0.6,0.4,0.2$, respectively. Therefore, the flow separation delayed with decreasing shear-thinning tendency (or with increasing values of the power-law indices). Similarly to the case with Newtonian fluids, the recirculation regions begin to occur beyond a critical limit. Figs. 6-8 show that the size of the recirculation zone also increases with a decrease in power-law index from 0.6 to 0.2 . This is due to the fact that as shear-thinning behavior increases the viscous forces decrease. Overall, from Figs. 5-8 it is notable that for the same Reynolds number the recirculation zones penetrate far much deeper into the side branch as the power-law index decreases. This is in good agreement with the flow patterns for Newtonian/non-Newtonian fluids as represented by Matos and Oliveira [18].

On the other hand, a secondary recirculation zone is also observed near the junction in the main branch as the fluid gets diverted into the side branch at different values of Re and $n$. This is because of the centrifugal force experienced by the fluid near the branch region where the fluid is forced to turn into the side branch. To represent it in a somewhat better way, the magnified views of streamline contours near the junction can also be seen in Figs. 5-8. For the range of conditions studied, the appearance of the secondary zone is observed for $n=1$ (at Re $\geqslant 150$ ), $n=0.6$ (at $\operatorname{Re} \geqslant 100$ ) and $n=0.4$ (at $\operatorname{Re}=200$ ). The intensity of the second- ary recirculation zone is weak here, but can be dominant in coiled ducts as centrifugal force is sustained in such geometries.

\subsection{Recirculation length}

The variation of the non-dimensional recirculation length $\left(L_{r} / D\right)$ in the side branch (defined as the distance from the junction of Tchannel to the point of attachment of the fluid with the bottom wall) as a function of Reynolds number and power-law index is shown in Fig. 9. The length of the recirculation zone is seen to increase in a non-linear fashion with an increase in Reynolds number for a particular power-law index. The recirculation length is also seen to increase with decreasing power-law index for a fixed Reynolds number. Thus, the dimensional considerations suggest that the recirculation length is a function of Reynolds number and power-law index. To correlate the dimensionless recirculation length with Reynolds number and power-law index, the data obtained is modelled to an exponential equation of the following form:

$L_{r} / D=a \operatorname{Re}^{b}+c$

where $a, b$ and $c$ are fitting coefficients and are given in Table 5. The maximum percentage deviation in the value of wake length from Eq. (4) with the present computed results is also indicated in Table 5.

\subsection{Onset of flow separation in the side branch}

The critical value of the Reynolds number at which the onset of recirculation zone begins to appear in the side branch is of great importance. This critical value of Reynolds number is determined for a range of values of the power-law indices studied here. This was accomplished by gradually increasing the Reynolds number in fixed increments for a particular power-law index while for each

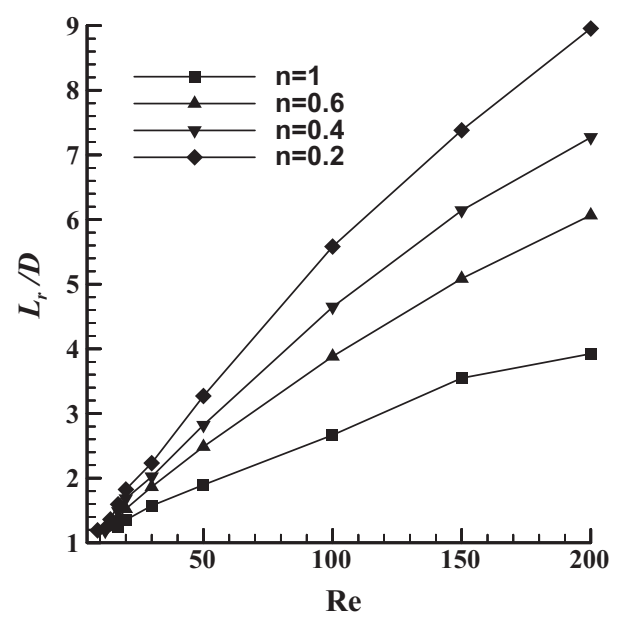

Fig. 9. Variation of dimensionless recirculation length $\left(L_{r} / D\right)$ with Reynolds number at different values of power-law index.

Table 5

Coefficients of exponential fit (in Eq. (4)) for the variation of dimensionless recirculation length with Reynolds number and power-law index.

\begin{tabular}{lllll}
\hline$n$ & $a$ & $b$ & $c$ & \% Maximum deviation \\
\hline 1 & 0.0949 & 0.6744 & 0.6120 & 4.2 \\
0.6 & 0.1374 & 0.7041 & 0.3623 & 1.9 \\
0.4 & 0.1830 & 0.6926 & 0.1612 & 3.8 \\
0.2 & 0.2044 & 0.7142 & 0.0148 & 4.0 \\
\hline
\end{tabular}


(a)

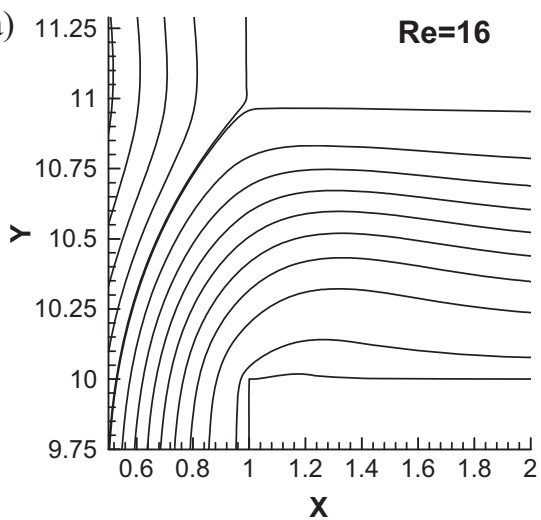

(b)

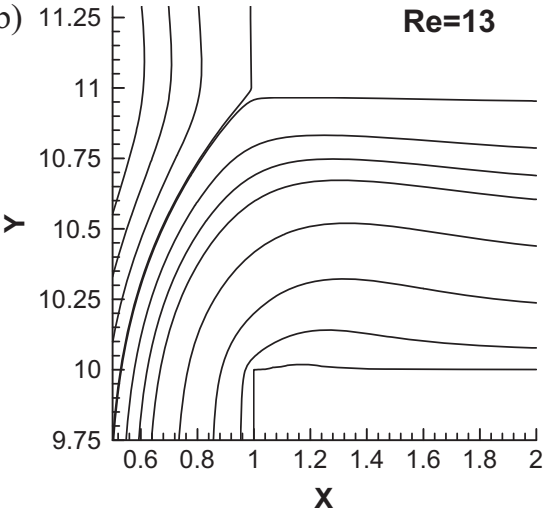

(c)

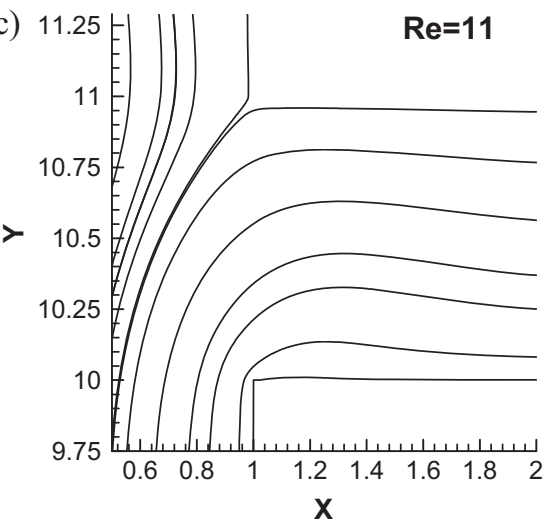

(d)

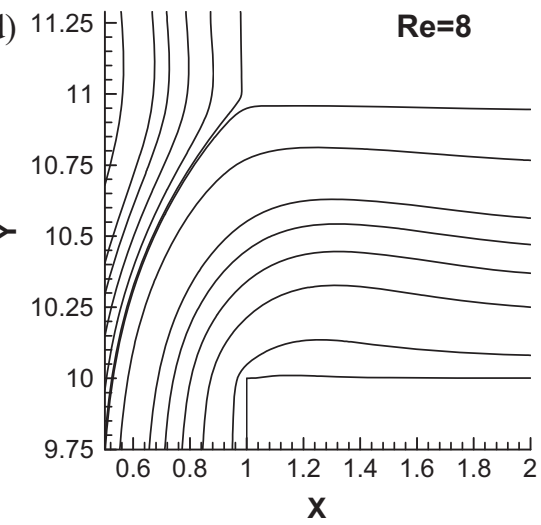

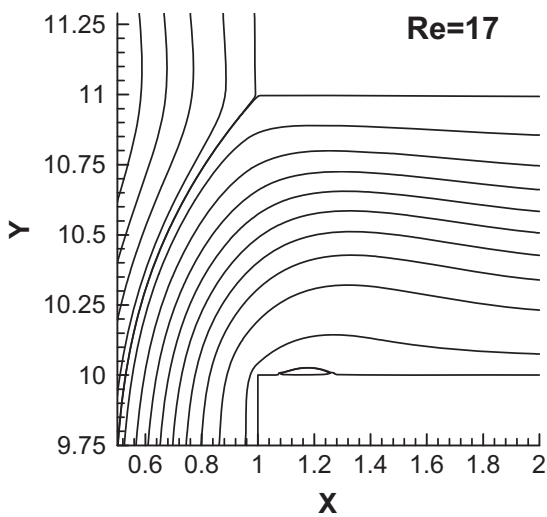

$\operatorname{Re}=14$

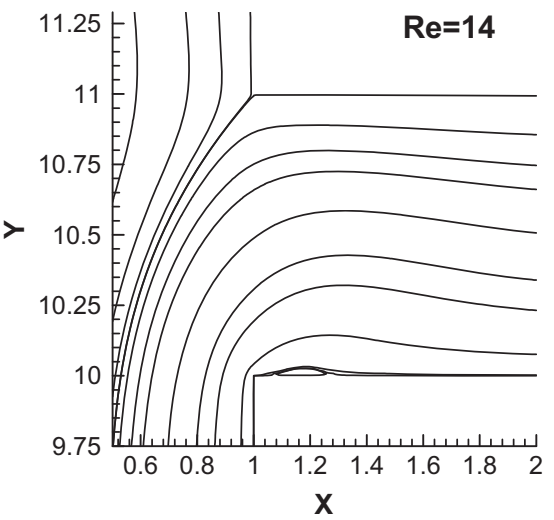

$\mathrm{Re}=12$
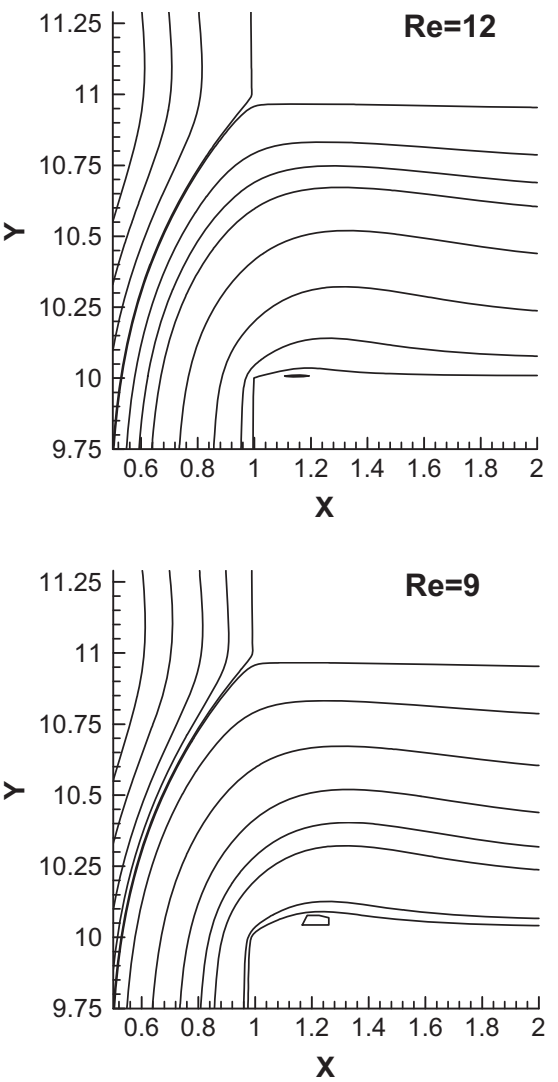

run the sign change in $X$-velocity on the lower wall of the side branch was monitored. A sign change indicates the existence of a recirculation zone in that region. The search was further refined by starting with the lower Reynolds number and repeating the search in smaller increments of Reynolds number. The critical Reynolds number was thus resolved to be within \pm 1 . Fig. 10 (a)-(d) 
shows the flow patterns in a T-channel for the critical Reynolds number at which the onset of flow separation occurs for the cases of $n=1,0.6,0.4$ and 0.2 , respectively. For the case of Newtonian fluid, no recirculation region appears in the side wall up to $\operatorname{Re}=16$, but as the Reynolds number is further increased to $\operatorname{Re}=17$, wakes begin to appear in the side branch. This indicates the critical value of Reynolds number for the case of Newtonian

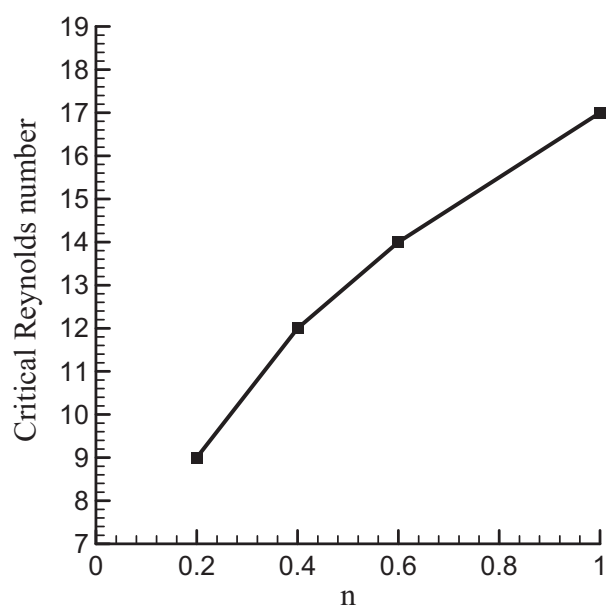

Fig. 11. Critical Reynolds number for the onset of flow separation at different values of power-law index. fluid to be $R e=17$. The critical Reynolds number for the case of Newtonian fluids is found to be in good agreement with the experimental results presented by Karino et al. [37] and Karino and Goldsmith [38]. Similar computations were performed on shearthinning fluids for examining the onset of flow separation at different values of $n$. For $n=0.6$, the fluid maintains its contact with the lower wall of side branch and no wakes are observed till $\operatorname{Re}=13$, but as we further carried our computations on $\mathrm{Re}=14$ a recirculation region begin to appear in the side branch. Similarly, for $n=0.4$ and 0.2 , no recirculation regions exist till $R e=11$ and $R e=8$ but begin to appear at $\operatorname{Re}=12$ and $\operatorname{Re}=9$, respectively. Fig. 11 shows the variation of the critical Reynolds number with power-law index. The Reynolds number at which the onset of flow separation takes place in the side branch decreases with the decrease in the power-law index, implying early recirculation zones with greater shear-thinning behavior.

\subsection{Variation of viscosity along the side branch}

The variation of viscosity along the bottom wall of a side branch of the T-junction for $\operatorname{Re}=5,50,100$ and 200 for three different values of $n(0.2,0.4$ and 0.6$)$ is shown in Fig. 12. These viscosity values are plotted for the first cells of the mesh away from the bottom wall. For the case of $\operatorname{Re}=5$, no recirculation (i.e., the wake length is zero here) exists in the flow patterns for any value of the power-law index, as seen from Figs. 5-8. However, a peak in the value of the power-law viscosity near the entrance of the side branch for $\mathrm{Re}=5$ is seen in Fig. 12, which is due to the small centrifugal force experienced by the fluid in this region. For the cases of $\operatorname{Re}=50,100$ and 200, the recirculation zones exist in the flow
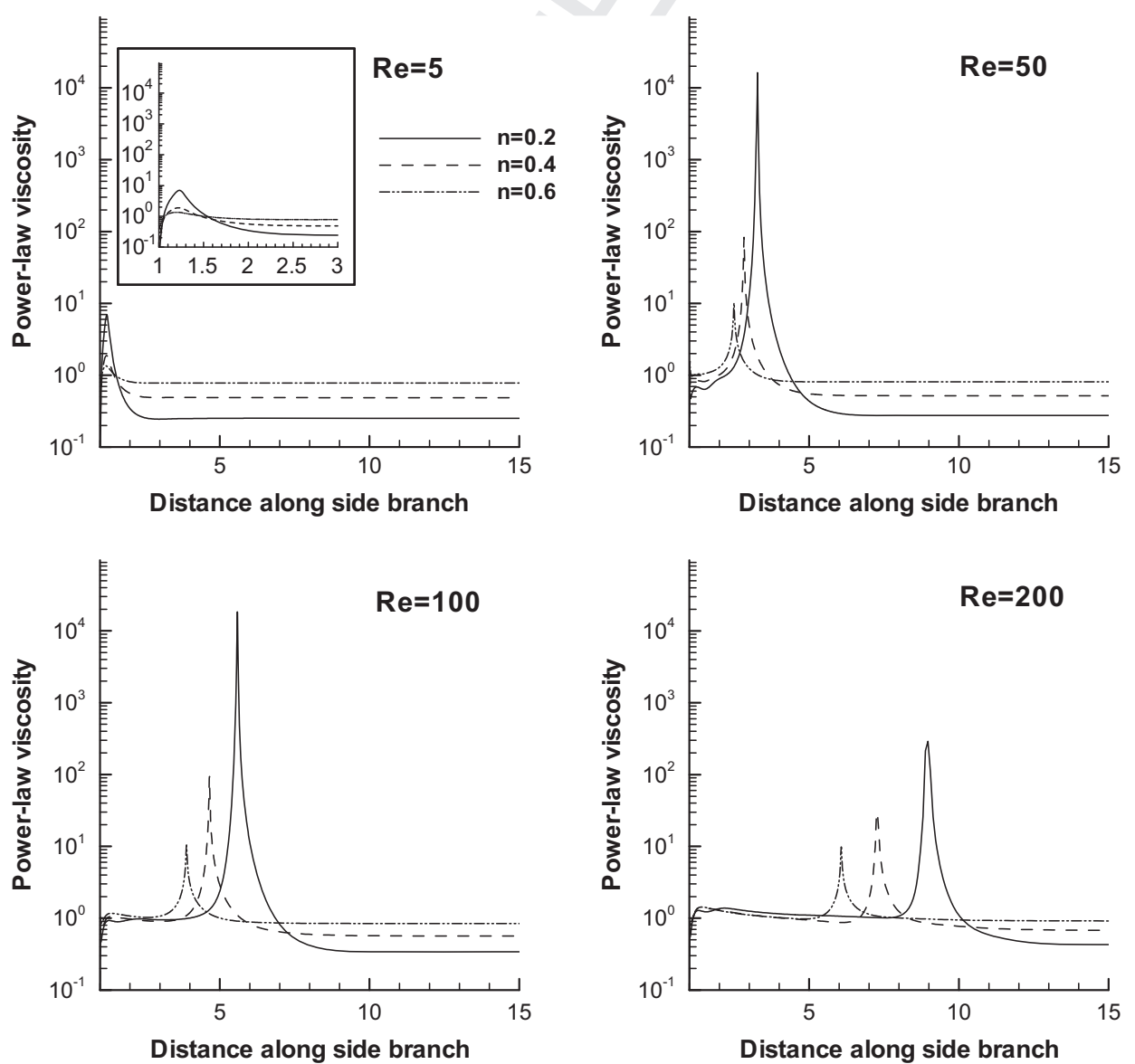

Fig. 12. Variation of power-law viscosity along the lower wall of side branch for $\operatorname{Re}=5,50,100$ and 200 at different values of $n$. 
patterns of the side branch (Figs. 5-8). A maximum is observed in the values of power-law viscosities in the bottom wall of the side branch, which then decreases to a constant value further along the side branch. As expected, the maximum/peak viscosity decreases with increasing power-law index at a fixed value of Reynolds number. The maximum values of viscosities at the bottom wall of the side branch appear at their wake lengths (or at the right end of the wake region) for different values of Reynolds numbers and power-law indices. For the case of Newtonian fluids, the viscosity of the fluid remains constant throughout its span in the T-junction.

\section{Conclusions}

In the present work, the effects of Reynolds number and powerlaw index on the flow of power-law fluids through a T-channel have been investigated. The flow is found to be steady for the entire range of settings investigated here. The numerical methodology has been extensively validated against previous numerical and experimental studies. The grid and computational domain were chosen after extensive testing by varying various grid and domain sizes. Detailed observations of flow pattern, recirculation length, critical Reynolds number for the onset of flow separation and viscosity variation along the bottom wall of the side branch have been presented. The results show that the length of the recirculation zone increases on increasing Reynolds number for a particular power-law index. It also increases on decreasing the power-law index for a fixed Reynolds number. The critical Reynolds number at which the onset of flow separation takes place in the side branch decreases with the decrease in the power-law index (or with increasing shear-thinning tendency). The maximum values of viscosities at the bottom wall of the side branch appear at their wake lengths for different values of Reynolds numbers and power-law indices. Furthermore, as the flow disturbances in shear-thinning fluids are much more dominant and exist up to a larger distance in the side branch than in Newtonian fluids, then to compute those disturbances one needs to have a fine mesh to a longer distance.

Although, the current work involves a numerical investigation into the problem of 2-D laminar flow of power-law shear-thinning fluids in a T-channel, subsequent experimentation of the same is necessary by using one or the other commercial flow measurement techniques viz. Laser Doppler Velocimetry, Particle Image Velocimetry and others. It is also advisable to perform 3-D simulations for capturing the accurate flow characteristics of highly shear-thinning fluids in a T-channel at Reynolds number higher than that used in this study. This is because the 2-D numerical simulations may be deemed inadequate to simulate the actual 3-D behavior of the shear-thinning fluids at high Reynolds numbers because of the remarkable change in their flow characteristics. Besides this, the same analysis can be performed on T-shaped bend tubes for the analysis of pressure drop and recirculation zones, as done in [24]. In addition to the onset of flow separation, it is also advisable to study the onset of instability in Newtonian and non-Newtonian fluids. This could be performed by some new methods for instability analysis such as the energy gradient method $[19,20]$ or semi-analytical method [39]. Furthermore, a heat transfer study may be performed for the present flow system.

\section{Acknowledgements}

The authors would like to thank the two anonymous reviewers for their valuable and helpful comments on this work.

\section{References}

[1] Liepsch D, Moravec S, Rastogi AK, Vlachos NS. Measurements and calculation of laminar flow in a ninety degree bifurcation. J Biomech 1982;15:473-85.

[2] Khodadadi JM, Nguyen TM, Vlachos NS. Laminar forced convective heat transfer in a two-dimensional $90^{\circ}$ bifurcation. Numer Heat Transfer 1986;9:677-95.

[3] Grace JL, Priest MS. Division of flow in open channel junctions. Bulletin No. 31 Engineering Experimental Station, Alabama, Polytechnic Institute, Auburn, Ala; 1958.

[4] Hayes RE, Nandakumar K, Nasr-El-Din H. Steady laminar flow in a 90 degree planar branch. Comput Fluids 1989;17:537-53.

[5] Neary VS, Sotiropoulos F. Numerical investigation of laminar flows through 90degree diversions of rectangular cross-section. Iowa Institute of Hydraulic Research, The University of Iowa, Iowa City, IA 52242, USA; 1995.

[6] Weber LJ, Schumate ED, Mawer N. Experiments on flow at a $90^{\circ}$ open-channel junction. J Hydraul Eng 2001;127:340-50.

[7] Yanase S, Kaga Y, Daikai R. Laminar flows through a curved rectangular duct over a wide range of the aspect ratio. Fluid Dyn Res 2002;31:151-83.

[8] Huang J, Weber LJ, Lai YG. Three-dimensional numerical study of flows in open-channel junctions. J Hydraul Eng 2002;128:268-80.

[9] Ramamurthy AS, Junying Qu, Diep Vo. Numerical and experimental study of dividing open channel flows. J Hydraul Res 2007;133:1135-44.

[10] Shamloo H, Pirzadeh B. Investigation of characteristics of separation zones in T-Junctions. WSEAS Trans Math 2008;7:303-12.

[11] Bramley JS, Dennis SCR. The numerical solution of two-dimensional flow in a branching channel. Comput Fluids 1984;12:339-55.

[12] Dennis SCR, Hudson JD. A difference method for solving the Navier-Stokes equations. In: Proc 1st conf num meth laminar turbulent flow. London: Pentech Press; 1978. p. 69.

[13] Ehrlich LW, Friedman MH. Computational aspects of aortic bifurcation flows. Comput Fluids 1985;13:177-83.

[14] Miranda AIP, Oliveira PJ, Pinho FT. Steady and unsteady laminar flows of Newtonian and generalized Newtonian fluids in a planar T-junction. Int J Numer Methods Fluids 2008;57:295-328.

[15] Khodadadi JM, Vlachos NS, Liepsch D, Moravec S. LDA measurements and numerical prediction of pulsatile laminar flow in a plane 90-degree bifurcation. J Biomech Eng 1988;110:129-36.

[16] Khodadadi JM. Wall pressure and shear stress variations in a 90-deg bifurcation during pulsatile laminar flow. J Fluids Eng 1991;113:111-5.

[17] Moshkin N, Yambangwi D. Steady viscous incompressible flow driven by a pressure difference in a planar T-junction channel. Int J Comput Fluid Dyn 2009;23:259-70.

[18] Matos HM, Oliveira PJ. Steady and unsteady non-Newtonian inelastic flows in a planar T-junction. Int J Heat Fluid Flow 2013;39:102-26.

[19] Hajmohammadi MR, Nourazar SS, Campo A. Analytical solution for two-phase flow between two rotating cylinders filled with power law liquid and a micro layer of gas. J Mech Sci Tech 2014;28:1849-54.

[20] Hajmohammadi MR, Nourazar SS. On the insertion of a thin gas layer in microcylindrical Couette flows involving power-law liquids. Int J Heat Mass Transfer 2014;75:97-108.

[21] Eesa M, Barigou M. CFD analysis of viscous non-Newtonian flow under the influence of a superimposed rotational vibration. Comput Fluids 2008;37:24-34.

[22] Turkyilmazoglu M. Dual and triple solutions for MHD slip flow of nonNewtonian fluid over a shrinking surface. Comput Fluids 2012;70:53-8.

[23] Hu B, Kieweg SL. The effect of surface tension on the gravity-driven thin film flow of Newtonian and power-law fluids. Comput Fluids 2012;64:83-90.

[24] Hajmohammadi MR, Eskandari H, Saffar-Avval M, Campo A. A new configuration of bend tubes for compound optimization of heat and fluid flow. Energy 2013;62:418-24.

[25] Csizmadia P, Hös C. CFD-based estimation and experiments on the loss coefficient for Bingham and power-law fluids through diffusers and elbows. Comput Fluids 2014;99:116-23.

[26] Bird RB, Stewartm WE, Lightfoot EN. Transport phenomena. 2nd ed. New York: John Wiley \& Sons Inc.; 2002.

[27] Chhabra RP, Richardson JF. Non-Newtonian flow and applied rheology. 2nd ed. Oxford (UK): Butterworth-Heinemann; 2008.

[28] Spelt PDM, Selerland T, Lawrence CJ, Lee PD. Flows of inelastic non-Newtonian fluids through arrays of aligned cylinders. Part 1. Creeping flows. J Eng Math 2005;51:57-80.

[29] Butcher TA, Irvine Jr TF. Use of the falling ball viscometer to obtain flow curves for inelastic, non-Newtonian fluids. J Non-Newtonian Fluid Mech 1990;36:51-70.

[30] Graham DI, Jones TER. Settling and transport of spherical particles in powerlaw fluids at finite Reynolds number. J Non-Newtonian Fluid Mech 1994;54:465-88.

[31] D’Alessio SJD, Pascal JP. Steady flow of a power-law fluid past a cylinder. Acta Mech 1996;117:87-100.

[32] Soares AA, Ferreira JM, Chhabra RP. Flow and forced convection heat transfer in cross flow of non-Newtonian fluids over a circular cylinder. Ind Eng Chem Res 2005;44:5815-27.

[33] Dhiman AK, Chhabra RP, Eswaran V. Steady flow of power-law fluids across a square cylinder. Chem Eng Res Des 2006;84:300-10. 
[34] Bharti RP, Chhabra RP. Two-dimensional steady poiseuille flow of power-law fluids across a circular cylinder in a plane confined channel: wall effects and drag coefficients. Ind Eng Chem Res 2007;46:3820-40.

[35] Reid WH. On the stability of viscous flow in a curved channel. Proc Roy Soc Lond 1958;A244:186-98.

[36] Roache PJ. Verification and validation in computational science and engineering. Albuquerque, New Mexico: Hermosa Publishers; 1998.
[37] Karino T, Kwong HHM, Goldsmith HL. Particle flow in models of branching vessels: I. Vortices in 90 T-junctions. Biorheology 1979;16:231-48.

[38] Karino T, Goldsmith HL. Particle flow in models of branching vessels: II. Effects of branching angle and diameter ratio on flow patterns. Biorheology 1985;22:87-104.

[39] Hajmohammadi MR, Nourazar SS. On the solution of characteristic value problems arising in linear stability analysis; semi analytical approach. Appl Math Comput 2014;239:126-32. 